\title{
Lateral Deformation of Sliver in Roller Drafting Zone
}

\author{
By Masaaki Okamura, Shinya Kurosaki, Fujio Konda \\ and Tatsuharu Ikeguchi, Members TMSJ
}

\author{
Faculty of Engineering, Gifu University, Yanagido, Gifu
}

Based on the Journal of the Textile Machinery Society of Japan, Transactions, Vol.43, No.3, T19-T25 (1990-3)

\begin{abstract}
The drafting force has been only the way to evaluate the aspects of roller drafting up to now. The lateral deformation (LD) of sliver would be newly proposed as a significant expression of the drafting behavior adding to the conventional drafting force.

Generally, the LD curve shows a high negative correlation to both the drafting force curve and the irregularity curve of output sliver. The mean, the coefficient of variation and the power spectrum of the LD curves were calculated, and the effects of the drafting conditions on these three factors were discussed. It becomes clear that the coefficient of variation of the LD shows the same tendency as that of the drafting force on the drafting conditions. The LD could be considered as one of the most important expressions to show the roller drafting aspects.
\end{abstract}

\section{Introduction}

In tensile test of the sliver, the tension increases first, shows a peak and then decreases. The sliver contracts while the tension increases, but on the contrary it expands while the tension decreases ${ }^{[1]}$. Such contraction and expansion are important properties of sliver, which are called the lateral deformation (LD) of sliver in this research. The deformation occurs not only when the sliver is drawn but also when it is roller-drafted. S. Backer [2],[3] also observed this kind of the deformation in the roller drafting zone and emphasized its importance in the drafting phenomena.

In this paper, the lateral deformation of sliver in the drafting zone is proposed as a visual expression of the drafting behavior and its general properties and significance have been investigated.

\section{Experimental Method}

The scheme of the measuring apparatus is shown in Fig. 1(a). Two pairs of the light projector and the light receiver are set in the position of $15 \mathrm{~mm}$ from the front roller in two-line type roller drafting machine. As shown in Fig. 1(b), one pair $\left(C 1\right.$ and $\left.C 1^{\prime}\right)$ is set in the vertical direction and the other pair $\left(C 2\right.$ and $\left.C 2^{\prime}\right)$ is set in the horizontal direction. The photoelectric device is used for the light receiver. A slit $(50 \mathrm{~mm} \times 1 \mathrm{~mm})$ is opened on the surfaces of each of the light projector and the light receiver. This apparatus can simultaneously measure both the drafting force and the LD. The drafting force is detected by the strain-gauge $(E)$. Output signals from the two light receivers and the strain-gauge are amplified by

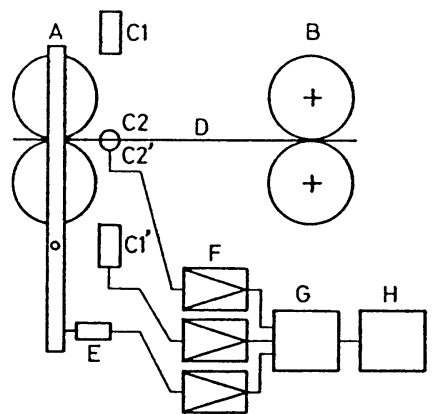

(a)

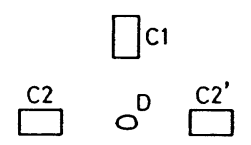<smiles>C1CC[Si]C1</smiles>

(b)

Fig. 1 (a) Measuring apparatus

A: Front roller B: Back roller C1, C2: Light projector $\mathrm{C1}^{\prime}, \mathrm{C}^{\prime}$ : Light receiver (Cds) $\mathrm{D}$ : Sliver E: Strain-gauge F: Amplifier G: A/D converter H: Micro computer

(b) Position of light receivers to sliver

the amplifiers $(F)$, pass through the A/D converter $(G)$ and are input into the micro computer $(H) .1024$ data per $102.4 \mathrm{sec}$ are obtained in an experiment. The drafting machine speed is $5 \mathrm{~m} / \mathrm{min}$. The material used is viscose rayon $(1.7 \mathrm{~d}, 64 \mathrm{~mm})$ card sliver. 


\section{Results and Discussion}

The LD and the drafting force were simultaneously measured at draft 10 and roller gauge $100 \mathrm{~mm}$. The output sliver irregularity was measured by the capacitance type tester. The results are shown in Fig. 2. The drafting force curve is very similar to the output sliver irregularity curve as well known ${ }^{[4]}$. The $\mathrm{LD}_{\mathrm{T}}$ (LD of sliver thickness) curve shows small values where the drafting force curve and the output sliver irregularity curve are large, and on the contrary shows large values where they are small. The $\mathrm{LD}_{\mathrm{W}}$ (LD of sliver width) curve shows little variation.

The correlation diagram of the drafting force and the output sliver weight is shown in Fig. 3(a). The correlation coefficient $r$ is 0.97 . As already known, they show

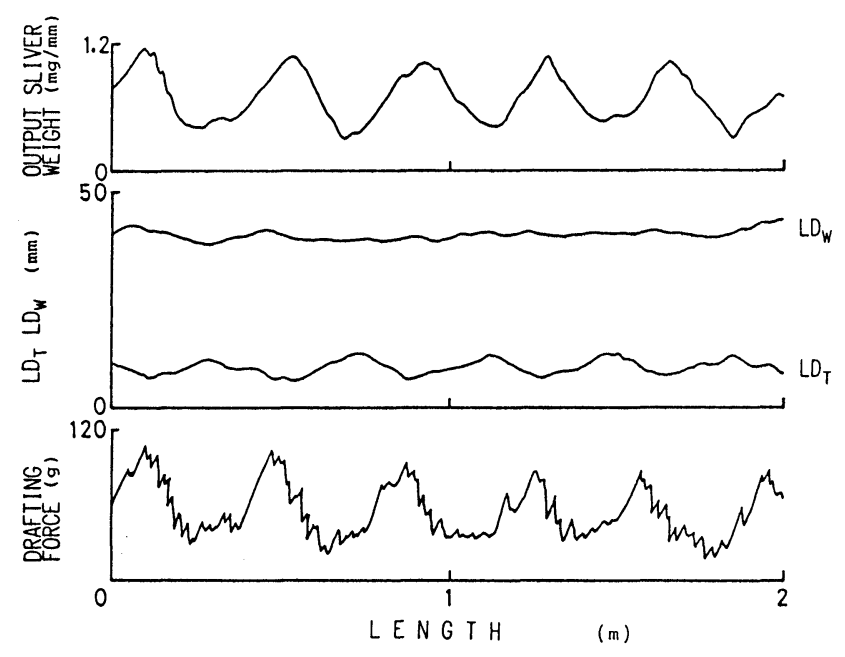

Fig. 2 Output sliver irregularity, $\mathrm{LD}_{\mathrm{W}}, \mathrm{LD}_{\mathrm{T}}$ and drafting force (Draft 10, roller gauge $100 \mathrm{~mm}$ )

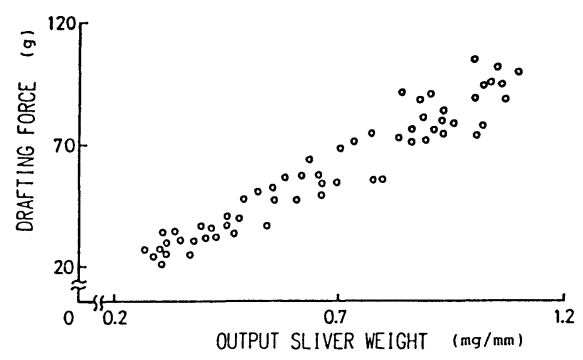

(a) Drafting force and output sliver weight

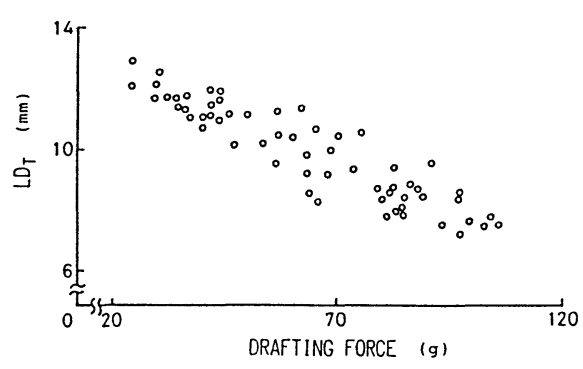

(c) LDT and drafting force high positive correlation. The correlation diagram (b) of the $\mathrm{LD}_{\mathrm{T}}$ and the output sliver weight, and (c) of the $\mathrm{LD}_{\mathrm{T}}$ and the drafting force show negative correlations together $(r=-0.91$ and $r=-0.89)$. The correlation diagram (d) of the $\mathrm{LD}_{\mathrm{W}}$ and the output sliver weight shows little correlation $(r=0.07)$. From these results, the $\mathrm{LD}_{\mathrm{T}}$ represents the $\mathrm{LD}$ in this research.

The LD curves and the drafting force curves of the other four kinds of sliver are shown in Fig. 4. The correlation coefficients of both LD and drafting force concerning with each sliver are shown as follows: -0.87 in cotton card sliver (a), -0.92 in acrylic card sliver (b), -0.88 in wool top (c), and -0.94 in polyester card sliver (d).

The LD and the drafting force were measured by varying the drafting conditions. In the case of the draft

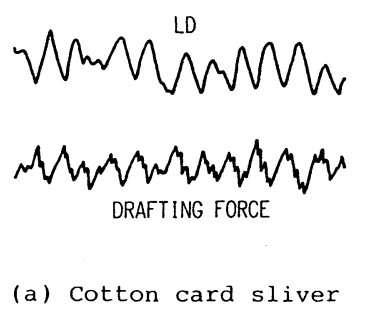

Munurums

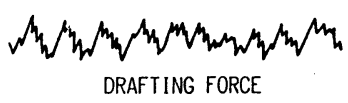

(c) Wool top sliver

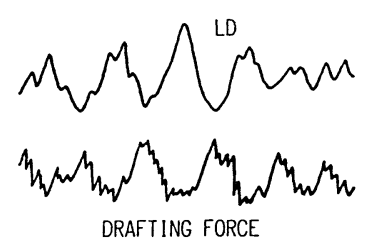

(b) Acrylic card sliver
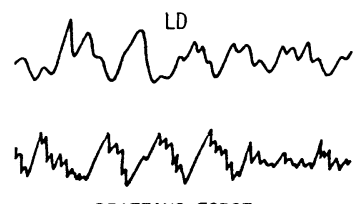

DRAFTING FORCE

(d) Polyester card sliver
Fig. 4 LD and drafting force for four slivers

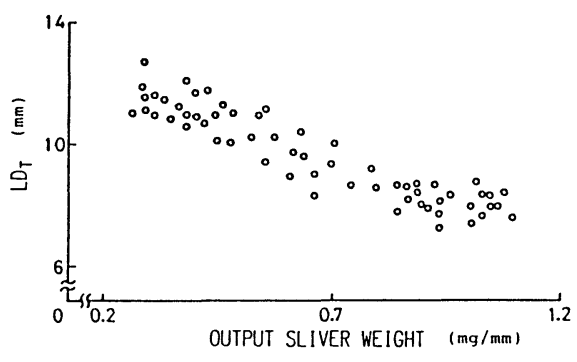

(b) LDT and output sliver weight

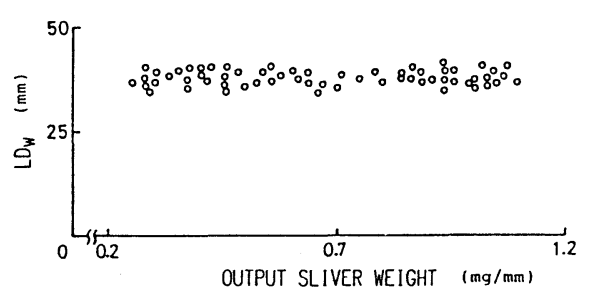

(d) LDW and output sliver weight

Fig. 3 Correlation diagrams 

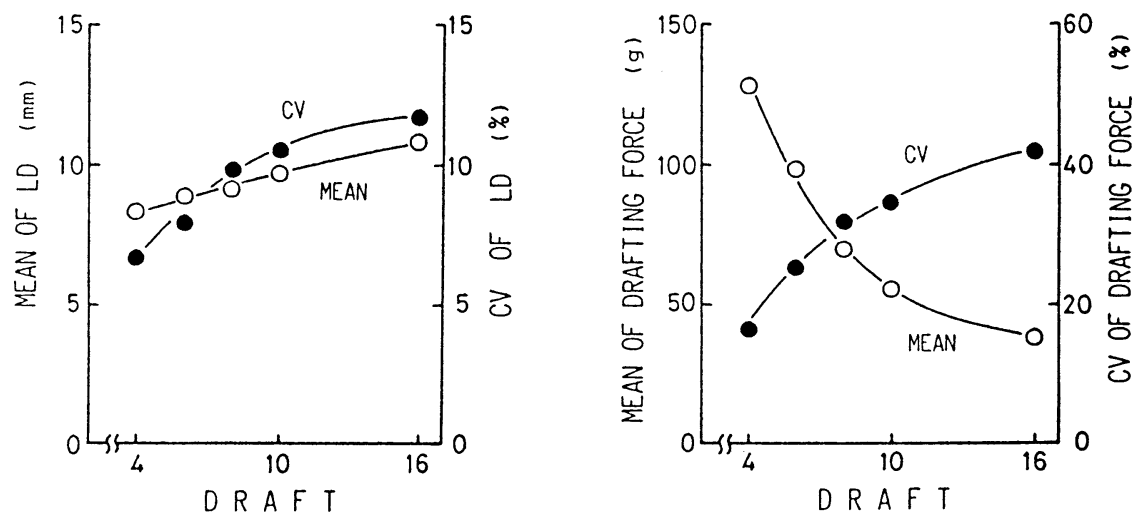

Fig. 5 LD and drafting force for draft

(Roller gauge $100 \mathrm{~mm}$ )

varied with the roller gauge $100 \mathrm{~mm}$, the mean and the coefficient of variation of both $\mathrm{LD}$ and drafting force are shown in Fig. 5. Both the mean and the coefficient of variation of the LD increases with the increase of the draft. While the mean of the LD shows a reverse tendency against the drafting force, the coefficient of variation increases in accordance with the draft.

The power spectrum of the LD curve was calculated. The power spectra of the LD curves for four drafts are shown in Fig. 6. The power spectrum has a peak in any draft. The peak becomes higher with the increase of the

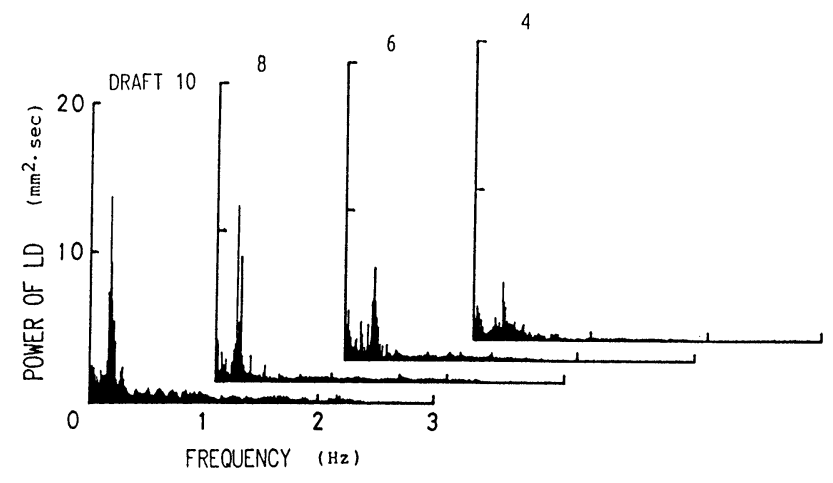

Fig. 6 Power spectra of LD for draft (Roller gauge $100 \mathrm{~mm}$ )

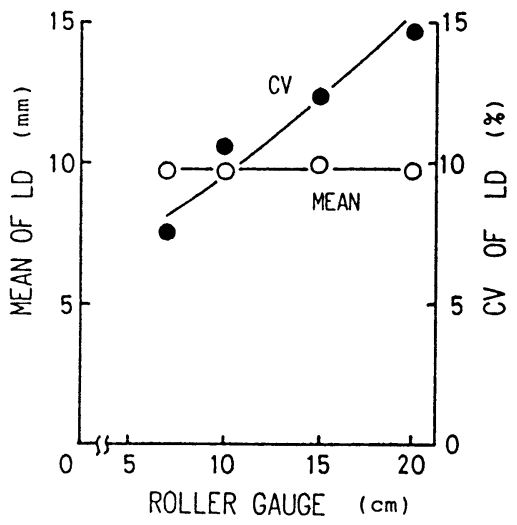

draft. This tendency means that the periodicity of the LD comes to be remarkable.

When the roller gauge is varied with draft 10 , the results are shown in Fig. 7 . With the increase of the roller gauge, the mean of the LD little varies and the coefficient of variation increases quickly, whereas the mean of the drafting force decreases while the coefficient of variation increases.

Figure 8 is the power spectra of the LD curves for four roller gauges. The periodicity of the LD increases with the increase of the roller gauge.

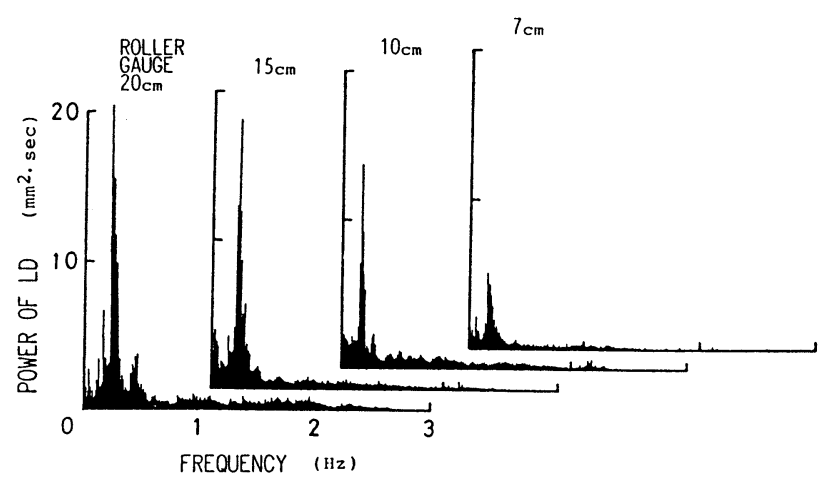

Fig. 8 Power spectra of LD for roller gauge (Draft 10)

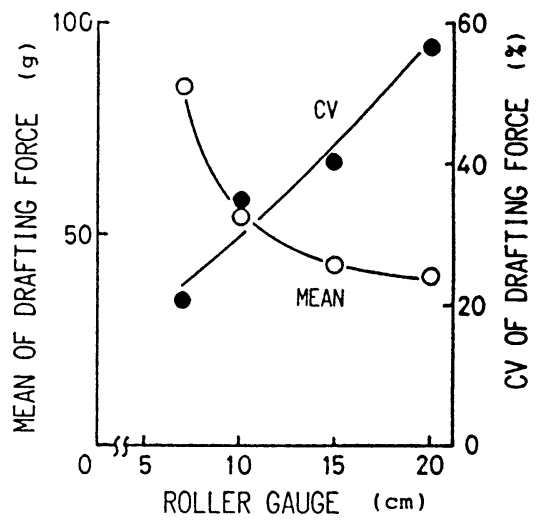

Fig. 7 LD and drafting force for roller gauge (Draft 10) 

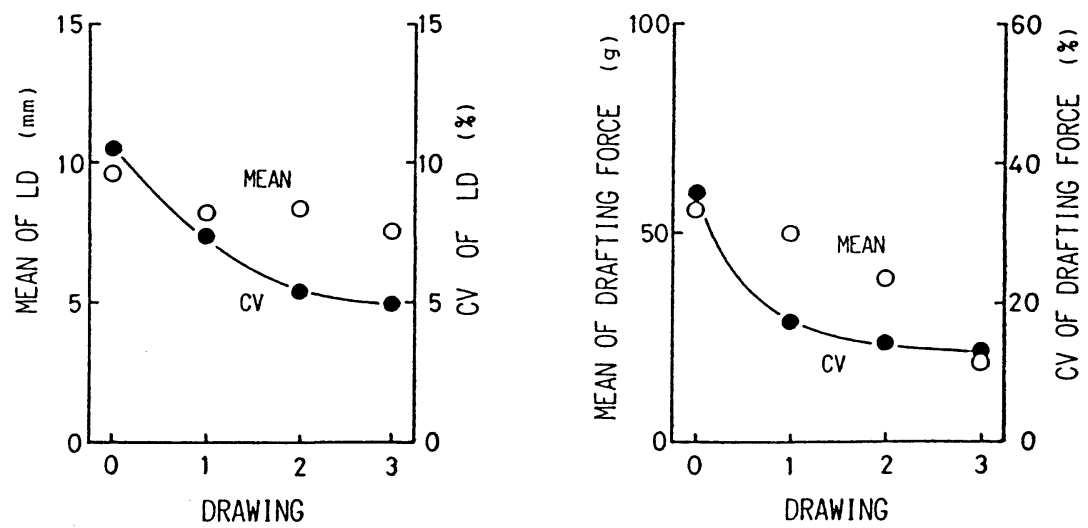

Fig. 9 LD and drafting force for drawing process (Draft 10 , roller gauge $100 \mathrm{~mm}$ )

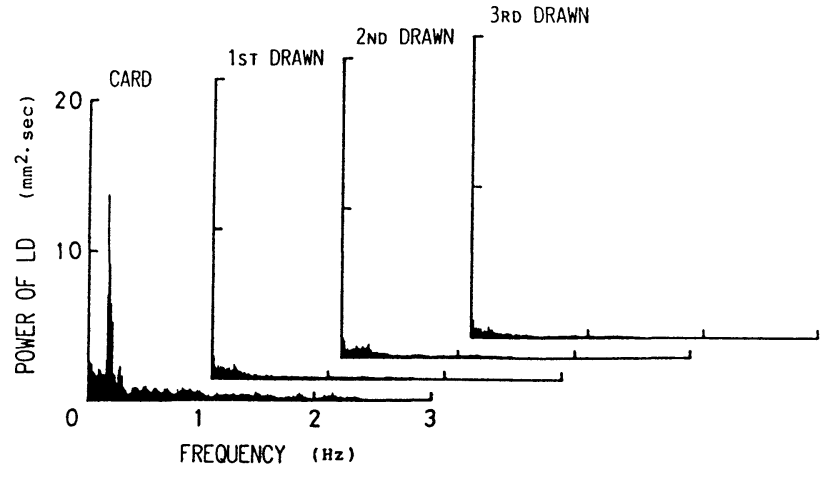

Fig. 10 Power spectra of LD for drawing process (Draft 10, roller gauge $100 \mathrm{~mm}$ )

Figure 9 is the results of the LD and the drafting force for drawing process. The coefficient of variation of the LD decreases with the process and shows the same tendency as the coefficient of variation of the drafting force.

The power spectra of the LD curves for the drawing process are shown in Fig. 10. In the card sliver, the LD shows a remarkable periodicity, but no periodicity of the LD is recognized in three kinds of drawing sliver.

\section{Conclusion}

The general properties and significance of the lateral deformation of sliver in the roller drafting zone have been investigated. The results obtained are as follows: (1) The LD curve shows high negative correlation with the drafting force curve and the output sliver irregularity curve.

(2) With the increase of the draft, both the mean and the coefficient of variation of the LD increase. The peak of the power spectrum of the LD curve becomes high, showing the periodicity of the LD increases.

(3) With the increase of the roller gauge, the coefficient of variation of the LD increases quickly and the periodicity increases while the mean little varies.

(4) With the increase of the number of drawing head, the coefficient of variation of the LD decreases. The periodical variation of the LD existing in the card sliver disappears in the draw sliver.

(5) The coefficient of variation of the $\mathrm{LD}$ for the drafting conditions shows the same tendency as that of the drafting force. The LD could become a significant index representing the drafting behavior.

\section{References}

[1] S. Kurosaki, F. Konda and M. Okamura ; Int. Wool Text. Res. Conf., 5, IV-167 (1975)

[2] H. R. Plonsker and S. Backer ; Text. Res. J., 39, 673 (1969)

[3] H. R. Plonsker and S. Backer ; Text. Res. J., 39, 823 (1969)

[4] W. Wegener and H. Bechlenberg ; Rayon Zellwolle u. Andere Chemiefasern, 14, 78, 142 (1955) 